Ann. Abeille, 196r, 4 (4), 277-296.

\title{
TREIZE ANS DE RECHERCHES APICOLES A BURES-SUR-YVETTE. ESSAI DE SYNTHESE
}

\author{
R. CHAUVIN \\ Station de Recherches sur l'Abeille et les Insectes Sociaux, Bures-sur-Yvette (S. \& O.).
}

I apicoles de Bures-sur-Yvette, la tâche qui s'offrait à moi et à l'équipe de chercheurs (jui m'accompagnait pouvait paraître délicate. Tous les pays européens ou presque disposaient de laboratoires voués à l'étude des Abeilles; certains étaient fort connus, comme l'Institut du LIEBEFELL, dirigé alors par le Dr. MORGENTHALER; et le génie expérimental du Professeur Karl von Fr IScH avait couvert de gloire son laboratoire de Munich et sa fameuse revue, véritable B ible de l'Apidologie, "Zeitschrift für vergleichende Physiologie ». Quant aux chercheurs français, bien qu'ils aient publié un certain nombre de travaux sur l'A beille, intéressants mais déjà anciens, ils n'avaient jamais disposá d'une organisation co nvenable. D'autant plus que nous sortions à peino de la période de l'entre deux guerres, difficile pour les chercheurs français, dans leur ensemble. D'autre part, ma formation propre était celle d'un spécialiste du comportement des Insectes en général et de la sociologie animale; mais je m’étais surtout occupé des Fourmis et maintenant encore je garde beaucoup de tendresse aux fauves roux et minuscules qui se cachent dans leur nid de brindilles au fond des forêts. Mes recherches sur l'Abeille, faute de matériel et d'organisation appropriée, avaient été peu nombreuses.

Que faire donc? Quelle direction adopter? Tout le monde me disait : maintenant que vous voilà chargé du laboratoire des Abeilles, vous allez étudier leurs danses, comme von FRISCH? Et je répondais : sûrement pas, au grand étonnement de la plupart. C'est que la stratégie de la recherche est délicate; les sentiers de la science sont nombreux et divergents ; certains conduisent à des territoires bien défrichés depuis vingt ans par des hommes de génie comme von FrISCH, entourés d'une équipe prestigieuse ; il n'y a pas de profit à les suivre; on ne peut plus récolter que les miettes qu'ils veulent bien vous laisser. Mieux vaut prendre certaines routes à peine frayées; car malgré les innombrables travaux parus sur les Abeilles, il reste encore bien des domaines mal connus. J'essayai donc de mettre en place un plan d'attaque, et j'y fus beaucoup aidé par la compréhension de 1'Institut National de la Recherche Agronomique, qui ne s'est jamais démentie jusqu'à présent, et que je tiens à remercier.

J'ai eu la chance que mes principaux collaborateurs du début soient encore ceux d'à présent ; ils acceptèrent avec enthousiasme de former ce dont on entend parler 
si souvent en France et qu'on voit si rarement, une équipe visant le même but. Nous sommes parvenus assez loin, je crois ; mais c'est parce que nos démarches ont été coordonnées.

Les membres de l'équipe sont bien connus maintenant en France et à l'étranger. LoUveaux était le seul botaniste, recrue précieuse pour un laboratoire d'apiculture ; je le priai donc de s'intéresser aux rapports des Abeilles et des fleurs, et surtout à la récolte du pollen; Mlle PAIN prit en main la physiologie du développement ovarien chez l'ouvrière, puis chez la reine. Je gardai pour moi le comportement, aidé de LECOMTE, de VuILLAUME et de l'abbé DaRcheN. Quant à LAVIE, je pensais le diriger du côté de la pathologie des Abeilles, mais il lui arriva au cours de ses recherches une singulière aventure dont je parlerai tout à l'heure.

\section{LES RECHERCHES D'ORDRE TECHNOLOGIQUE E'T L'HISTOIRE DE IA STATION AU COURS DES PREMIÈRES ANNÉES}

Nous nous installâmes donc tant bien que mal au début et je fis entreprendre la formation d'un rucher. Disons tout de suite que c'est ce dernier point qui me donna le plus de mal. Je n'avais pas fait de prévisions assez larges; d'autre part, des intoxications répétées par les épandages insecticides sur colza ruinèrent à trois ou quatre reprises nos espoirs de développement apicole. I1 fallut la création de la Station expérimentale d'Apiculture de Montfavet, annexe de Bures, pour mettre fin à nos difficultés d'ordre apicole ; M. Trouverot, directeur central du département de Zoologie Agricole à l'INRA, contribua de toutes ses forces à la réalisation de cette annexe dont Louveaux traitera d'autre part.

Le probleme des insecticides fut une source de difficultés dans nos rapports avec une partie de la profession apicole, désespérée par les catastrophes qui dévastaient chaque année les ruchers. Nous sentions bien l'importance du problème et je fis exécuter diverses recherches sur la courbe de floraison du colza et la claustration des ruches. C'est alors que sous l'impulsion des producteurs d'oléagineux furent entrepris nos travaux sur le toxaphène et sa toxicité vis-à-vis des Abeilles. Je me souviens encore de cette époque héroïque. La situation se détériorait d'année en année; il fallait réussir. Or nous étions en octobre et nous aurions bien voulu que le printemps suivant voie la fin des intoxications. Nous avons tout improvisé ; nous qui ne savions rien de la physiologie végétale, nous avons essayé de forcer le colza à fleurir au mois de janvier et nous y avons réussi, et les Abeilles réactivées ont butiné leurs nectaires débordants ; et le toxaphène à des doses pourtant énormes ne les a pas tuées. Si bien que nous avons pu à temps faire interdire les autres insecticides très toxiques et que le cauchemar apicole a pris fin la même année. Il a fallu pour cela énormément de travail et traverser beaucoup d'angoisses; VUILLAUME, déjà docteur ès sciences pourtant, se couvrait de boue en allant sélectionner les plants de colza dans les champs. Cet effort, bien oublié des apiculteurs, a porté ses fruits, et c'est l'essentiel.

Je suivais en même temps une idée qui m'était chère depuis longtemps : il m'avait semblé que l'apiculture serait toujours en fâcheuse posture si elle n'exploitait qu'un seul produit, le miel. On connait bien d'autres substances provenant de la ruche et qui pourraient présenter quelque intérêt, non seulement le venin, mais encore la gelée royale, le pollen, la propolis, etc. Nous n'avions alors que des données 
éparses sur leur composition chimique et nous ne savions rien de leur action physiologique possible sur les animaux supérieurs : c'est précisément ce dernier point que je voulais étudier. Il fallait partir de rien ou peu s'en faut. Je fis d'abord constituer à Bures une animalerie de quelques milliers de souris, car nos crédits ne nous permettaient pas de travailler sur les rats. Mais comment rechercher l'action sur les Mammifères d'un corps dont on ignore à pau près tout? Après quatre ans de réflexions, de tâtonnements, d'expériences qui ne menaient pas à grand'chose, il m'a semblé qu'on pouvait tout au moins tenter les sondages suivants. I) l'action sur la croissance à différentes doses. 2) l'action sur le poids des organes qui permat de s'orienter ; par exemple le degré d'involution du thymus est un indice précieux. 3) l'effet sur le nombre et le pourcentage des éléments figurés du sang; ainsi que l'action éventuelle sur qualques-unes de ses constantes biologiques comme la glycémie. 4) l'action sur le coefficient d'utilisation digestive, étude facilitée par le fait que j'avais pu mettre au point un dispositif permettant d'administrer sans perte à une souris une alimentation pulvérulente, tout en récoltant à part les excréments et les urines. 5) l'action sur le comportement en général : activité spontanée, tonus musculaire, émotivité, etc.

Je ne veux pas dire naturellement qu'une telle façon d'aborder le sujet l'épuise, et il existe vingt autres manières de procéder. Je pense seulement qu'étant donné les moyens dont nous disposions, nous pouvions réaliser ainsi une exploration raisonnable de l'effet d'un produit inconnu. Ces recherches sont en plein développement. Elles ont abouti d'une part à une démonstration de l'intérêt de la gelée royale (traitement de la sénescence, euphorisant général) et du pollen (action sur l'intestin et la formule sanguine). Pour mesurer l'incidence pratique de ces recherches il peut être intéressant de noter qu'il a été vendu en une seule année plus de $800 \mathrm{~kg}$ de gelée, au moment où son prix dépassait 500 frs le gramme, et qu'en I959-60 vingt tonnes de pollen des Abeilles ont trouvé preneur à un minimum de I ooo frs le $\mathrm{kg}$. Malheureusement, la discipline nécessaire dans la commercialisation d'un produit nouveau n'a pas été respectée; certains abus même ont été commis; si bien que le laboratoire se verra forcé de prendre plus de précautions que par le passé, au cas où d'autres produits révéleraient des propriétés intéressantes.

\section{LA POLITIQUE VIS-A-VIS DE IA PROFESSION IT IA VUIGARISATION}

Nous nous préoccupions en même temps de nous faire connaître dans la profession apicole et de l'intéresser aux travaux de la Recherche. C'est une tâche très délicate : j'hésitais et j'hésite encore sur les meilleurs moyens de l'accomplir. Nous avons d'abord voulu toucher la quasi-totalité des apiculteurs, amateurs et professionnels à l'aide de conférences et d'articles publiés à peu près exclusivement daus le plus ancien des journaux apicoles de France et probablement du monde, "1'Apiculteur ", qui nous offrit aimablement l'hospitalité. Nous fîmes aussi pendant plusieurs années des cours réservés à une élite et qui duraient huit jours. Puis je conclus que les apiculteurs amateurs étaient d'origines trop diverses et que leur intérêt pour l'apiculture avait des motifs trop variés pour que nous puissions les toucher utilement. Il était sans doute plus sûr de rechercher le contact avec les professionnels qui disposent de 500 ruches ou davantage et qui vivent entièrement de l'apiculture. Je dois dire que nous n'avons pas remporté de succès bien net ni dans un cas ni dans l'autre; 
nous avons perçu rarement l'écho de nos cours et conférences ; quant à nos articles, l'opinion unanime les trouvait toujours trop compliqués.

Nous avons tenté aussi différentes enquêtes pour nous renseigner nous-mêmes et renseigner la profession sur son propre état et sur ses objectifs. Ces enquêtes ont toujours été accueillies très favorablement et on nous a promis de nombreuses réponses : elles n'ont jamais atteint ro p. Ioo du total des questionnaires distribués aux professionnels, et nous n'avons donc pu en tirer aucune conclusion. Ajoutons d'ailleurs qu'il n'existe et n'a jamais existé aucune statistique complète sur l'ensemble de la profession ; il y a probablement de 20 à 30 ooo ruches par département, mais personne ne peut l'affirmer.

Il existe, je crois, plus d'une raison à un état de choses aussi décourageant. Seule parmi toutes les professions agricoles, l'apiculture ne trouve nulle part d'école de formation professionnelle; car les quelque heures de cours qui se donnent çà et là sur les Abeilles ne peuvent en tenir lieu. Cela entraîne plusieurs conséquences ; d'abord la pauvreté de la littérature apicole en général, distribuée dans de trop nombreux journaux ; alors que les périodiques de vulgarisation agricole exposent sous une forme simplifiée les conclusions de travaux scientifiques sérieux, et ne remettent pas en cause le rôle des engrais, ou de la sélection ni l'utitité de l'agronomie en général, l'apiculture ignore encore une science apidologique pourtant très développée. La deuxième conséquence est l'absence du progrès technique; beaucoup conviennent parmi les apiculteurs que la seule et dernière grande découverte qui a vraiment révolutionné 1'apiculture consiste dans la ruche à cadres mobiles ; mais elle remonte à bien longtemps déjà. Les innovations techniques sont d'ailleurs souvent mal accueillies dans la profession. Dans le passé l'introduction du "mobilisme " a donné lieu de la part des "fixistes " à toutes sortes de controverses qui se sont poursuivies pendant des lustres. J'ai vécu une expérience analogue, lorsque j'ai voulu introđuire l'usage généralisé des pièges à pollen.

Mais il ne sert à rien de se plaindre et mieux vaut chercher les moyens de remédier à un état de choses peu satisfaisant. Je pense que notre technique a été mauvaise et nos collègues du Ministère de l'Agriculture, spécialistes de la vulgarisation, ont justement attiré mon attention là-dessus. La distance est trop grande entre des professionnels dont le niveau moyen est celui du certificat d'étude et les hommes de science ; les seconds ne savent pas parler aux premiers. Il faut un échelon intermédiaire de vulgarisateurs professionnels qui manque jusqu'à présent et qui serait lui, instruit directement par les hommes des laboratoires. En un mot, un système à deux degrés, élémentaire et supérieur, comblerait sans doute les très graves lacunes que j'ai signalées et remettrait en marche dans l'apiculture le progrès technique si nécessaire dans le monde moderne.

Comme le montre l'étude de Louve,aux et I, AVIE nous avons voulu servir autrement le progrès en prêchant d'exemple. On prépa re en France le miel comme on le faisait naguère pour le lait, sans hygiène parfois, sans les précautions techniques indispensables trop souvent. Les apiculteurs ignorent l'usage du réfractomètre pour la mesure de la teneur en eau, ne disposent pas de dispositifs pour la fonte du miel vraiment adéquats, etc... En Amérique au contraire, la technologie du miel est parfaitement connue et pratiquée. Des crédits importants nous ont été attribués par 1'INRA pour monter dans notre annexe de Montfavet une installation très complète de traitement du miel, la plus moderne d'Europe. A peine commencée, elle a suscité 1'enthousiasme 
un peu hâtif de certains professionnels et, à peine terminée, des critiques quelque peu irréfléchies. Mais voici qu'un peu partout se montent des coopératives (dont certaines, comme celle du Gâtinais, démarrent avec des moyens puisssants) et qui veulent traiter le miel à l'exemple de Montfavet. Elle découvrent du même coup l'existence de la Station de Bures, beaucoup plus adaptée à l'insertion dans une structure industrielle comme la leur qu'à se débrouiller au milieu des particularismes et des préjugés artisanaux. Peut-être, après tout, les temps sont-ils accomplis?

L'INRA nous a permis aussi de fonder sous son égide un journal d'apidologie, les "Annales de l'Abeille ", qui présente la masse sans cesse croissante des travaux du laboratoire et aussi quelques travaux étrangers. Cette formule a tous les avantages, car l'appui d'un service officiel nous permet de négliger les problèmes commerciaux de la diffusion d'un périodique. Nous pensions dès le début garder aux "Annales" un niveau scientifique élevé, sans employer trop systématiquement un langage abstrait. Nous espérions pouvoir sélectionner ainsi les quelques centaines d'apiculteurs français ou étrangers qui disposent de la culture nécessaire pour s'intéresser à la science de l'Abeille. Je dois dire que le succès des Annales a été immédiat et considérable ; il a dépassé nos prévisions les plus optimistes puisque le nombre des abonnés dépasse maintenant $45^{\circ}$ et que l'étranger s'y intéresse de plus en plus.

\section{LE PERSONNEL, FT I'ORgaNishtion DE I, STATION DE BURES FN I96I}

Actuellement (avril I96I) la Station de Bures dont j'assume la direction, comprend un maître de recherches (LOUVEAUX) deux chargés de recherches (LECOMTE et $M^{11}$ PAIN) et deux chercheurs associés depuis toujours à nos travaux, VUILLAUME, maître de conférences à la Faculté des Sciences de Nancy, et l'abbé DARCHEN, chargé de recherches du Centre National de la Recherche Scientifique. Le personnel scientifique a été renforcé récemment par POUVREAU et BARBIER, contractuels. Les hommes de science sont aidés par deux techniciens supérieurs Jouaur t et LouIs, cinq techniciens, Mlles Lhos'te, Chancerel, et S. Chauvin, MM. Paris et Chertier ainsi qu'un "Biennenmeister ", TheurkaurF. A cette équipe s'ajoutent un ouvrier spécialisé chargé de 1'atelier, Chardes, deux secrétaires et deux femmes de ménage.

On pourrait représenter ainsi l'organisation de la Station :

Comportement des abeilles $\left\{\begin{array}{l}\text { Chauvin } \\ \text { Lecomte } \\ \text { Vuili,Aume } \\ \text { DARCHen }\end{array}\right.$ Tech. : Paris, S. Chauvin

Biométrie, Botanique apicole, Miel .. $\begin{aligned} & \text { LOUveAux } \\ & \text { BARBIER }\end{aligned}$ Tech. : Louis, Lhoste

Technologie des produits de la ruche

autres que le miel ............ Chauvin 'Tech. : Douault, Chertier

Physiologie générale, Hormones .... M MIte PAIN Tech. : Chancerel

Pollinisation $\ldots \ldots \ldots \ldots \ldots \ldots \ldots\left\{\begin{array}{l}\text { LECOMTE } \\ \text { PouvreaU }\end{array}\right.$ Tech. : Paris, Theurkauff 
Ajoutons que d'assez nombreux stagiaires viennent tous les ans s'initier à la biologie des Abeilles ; certains reviennent régulièrement et entreprennent un travail de longue haleine : ainsi NAULLEAU, licencié ès sciences, qui étudie les transpositions de larves et leurs conséquences ; MONTAGNER et ROBER'T (sous la direction de VuilLAUME) s'attachent au développement des cellules royales et à la ponte de la reine. $M^{11}$ SALIERON rédige une thèse du troisième cycle sur les échanges entre Abeilles étudiés au moyen des radioisotopes; MHe PETrT et RIERA préparent le même diplôme, la première sur la croissance in vitro des larves d'Abeilles, la seconde sur le vieillissement des cires, étudié du point de vue chimique et histologique. D'autre part nous avons reçu un certain nombre de stagiaires étrangers parmi lesquels LENSKY (Israël) s'est intéreszé à la résistance desAbeilles à la chaleur et JoYAPPA (Inde) à la biométrie. Si bien que le personnel en pleine activité peut dépasser 25 personnes. Si l'on ajoute à cela les 8 personnes de notre annexe de Montfavet, on obtient 28 permanents, disposants de trois voitures automobiles et de 80 ooo $\mathrm{NF}$ de crédits de fonctionnement. Il s'agit donc d'un très gros effort consenti par l'I N R A. en faveur de l'apiculture. On peut noter que par ordre d'importance des moyens, le complexe Bures-Montfavet doit être le deuxièma du monde, dépassé seulement par le grand laboratoire russe de Rybnoe (province de Ryazan) dirigé par Gi,ustkov. D'autres pays, comme 1'Allemagne et l'Amérique, disposent de plus de chercheurs apicoles que nous mais ils sont très dispersés et parfois isolés.

\section{Les moyens de travail.}

Bures dispose d'un rucher d'environ 80 ruches uniquement destinées aux expériences et non à la production de miel (ce qui est réservé à Montfavet); d'une bibliothèque qui comprend trois sections : apiculture (avec 3 à 4 ooo tirés à part, photocopies et livres sur l'Abeille) ; éthologie générale (un laboratoire d'éthologie expérimentale de 1'École des Hautes Êtudes, est rattaché à la Station) entomologie générale et physiologie de 1'Insecte. Un colloque hebdomadaire réunit pendant la mauvaise saison tous les chercheurs et techniciens du laboratoire; chacun y expose ses recherches qui sont libéralement et parfois vigourausement critiquées par les autres. Pendant la belle saison les scientifiques et techniciens doivent bloquer en trois mois une grande quantité d'expériences et ne disposent plus d'assez de temps pour ces colloques. Le budget annuel y est discuté ainsi que sa répartition.

Je tiens beaucoup à ce que les cherchears ne s'enferment pas exclusivement dans la science apidologique et soient familiers avec d'autres problèmes : c'est pourquoi mes collaborateurs ont chacun plusieurs sujets d'étude. Je m'occupe moi-même en plus des Abeilles, du comportement des fourmis; LicomTE du comportement des araignées et des animaux supérieurs ; VUILLAUMk des guêpes ; DARCHEN de l'apprentissage des Insectes dans le labyrinthe, etc.

\section{LES RESULTATS SCIENTIFIQUES}

Ils comprennent comme on le verra dans la bibliographie, I63 travaux, trois thèses (LouvEAUX, DARChEN, LAVIE) déjà soutenues et trois autres (PAIN, LECOMTE, BARBIER) qui vont 1'être incessamment. Un traité de biologie de l'Abeille est en préparation, 
ainsi qu'un Manuel d'Apiculture. La synthèse de tous ces travaux se divise facilement en plusieurs chapitres.

\section{LE COMPORTEMFNT E'T LA BIOLOGIE DE LA RUCHE.}

Dès mon arrivée à la Station, après avoir repris certains phénomènes dus à "l'effet de groupe " qui commandent la survie des Abeilles à 1'état isolé, et que j'avais đéjà étudiés avec Grassé, je me suis intéressé aux moyens d'observation directe de la ruche. En effet l'ouverture d'une ruche à cadres mobiles pour observer ce qui s'y passe est une opération beaucoup plus grave qu'on ne le croit généralement; dans la mauvaise saison, comme BüDE, l'a montré, il suffit d'enlever seulement le couvre cadre et d'expédier une seule bouffée de fumée pour que la perturbation thermique qui s'ensuit mette plus de 24 heures à se calmer. J'aurais voulu disposer les colonies de telle sorte que l'on puisse apprécier chaque jour la ponte de la reine, l'activité générale, la qualité de la régulation thermique, la récolte du pollen et du nectar, etc. C'est évidemment la mesure de la ponte de la reine qui présente les plus grandes difficultés. J'ai eu la chance de les vaincre presque au début en collant sur une plaque de verre de la cire gaufrée sur une face seulement. Il est alors extrêmement curieux, en regardant par transparence du còté de la plaque de verre vierge de cire, de voir la reine en train de pondre : les coufs qui se déposent au fond des cellules sont très visibles, car les abeilles en étirant la cire laissent presque à nu le verre au fond de chaque cellule. On pent ainsi maintenir anssi longtemps que l'on veut des colonies en observation. On s'aperçoit alors de l'extrême sensibilité de la ponte aux incidences de la miellée et aussi des énormes différences de ponte entre denx reines voisines et de même race élevées cote à côte. Mais cette nuéthode présente des difficultés dont la principale est qu'il reste dificile d'obtenir des colonies d'une taille normale; le collage des plaques de cire sur des plaques de verre de grande dimension est en effet si dirricile que je n'ai pu le réaliser de manière satisfaisante. Aussi ai-je préféré depuis peu tun moyen terme qui semble parfaitement réussir : je réalise un très grand cadre de I $m \times$ I m à une seule face utilisable (l'autre étant si rapprochée de la vitre arrière (qu'elle permet tout juste le passage d'une Abeille). Il ne faut surtout pas que la cire touche le verre sinon les Abeilles l'enlèvent. Alors les ouvrières et la reine se cantonnent sur une seule des deux faces, la plus éloignée de la paroi. On obtient un développement régulier de la colonie, avec d'énormes plaques de couvain (l'une dépassait le demi-mètre carré!) à la condition toutefois que la ruche soit maintenue dans une armoire vitrée et thermostatée à $34^{\circ}$. Ces ruches unifaces présentent en effet, comme on peut s'en douter, une énorme déperdition thermique et si on ne leur fournit pas des calories supplémentaires, la colonie ne tarde pas à péricliter. Au moment de l'hivernage, on abaisse doucement la température aux environs de $20^{\circ}$. Je me suis aperçu que la possibilité de gouverner de l'extérieur l'état thermique de la ruche permet toute une série d'expériences très intéressantes.

Quant à l'activité, la bibliographie montre les grandes difficultés que présente sa mesure. On a essayé toutes sortes de dispositifs, généralement à contacts électriques, qui s'encrassent ou s'abîment très rapidement. J'ai monté moi-même un compteur à eau où les Abeilles poussaient pour sortir de petits leviers qui libéraient à chaque fois une goutte d'eau. Mais elles poussent les leviers plus ou moins vivement 
suivant la température, si bien que la quantité d'eau écoulée à un moment ne correspond pas à la même quantité d'Abeilles qu'à une autre période plus froide ou plus chaude. Les cellules photoélectriques sont d'un usage plus pratique malgré le prix des dispositifs d'enregistrement. 'T'out récemment enfin, j'ai pu réussir l'enregistrement d'une courbe d'activité par un dispositif purement mécanique; la quantité de pollen récoltée est enregistrée au-clessous ainsi que la transpiration de la ruche, c'est-à-dire la quantité d'eau rejetée lorsqu'il y a miellée (on la condense à l'aide d'un serpentin). Il est trop tôt pour interpréter toutes ces données, mais il est bien certain que nous ne progresserons réellement dans la biologie de la ruche que lorsque nous pourrons voir et mesurer tout ce qui s'y passe.

\section{IA CONSTRUCTION}

Il m'a semblé depuis toujours que les procédés de construction chez les animaux et en particulier chez les Insectes sociaux étaient l'indice de processus psychiques supérieurs auxquels appartient nécessairement 1'activité fabricatrice. Aussi ai-je orienté de ce côté les recherches de DARCHEN (cellules d'ouvrières) et de VUII_AUME (cellules de reines). Les résultats nous ont montré rapidement que nous explorions un champ de recherches particulièrement fécond.

La construction des rayons.

DARChen a écrit là-dessus de nombreuses notes et une thèse qui nous offrent de la construction une image assez différente de celle qu'on imaginait. Au début, il semble bien que la forme de l'essaim règle la disposition des ébauches de cire sur le substrat, sans doute par l'interaction de forces mécanicues assez simples oì la pesanteur joue le principal rôle. Ces ébauches sont ultérieurement remaniées et l'une finit par dépasser notablement les autres pour constituer le premier rayon ; c'est à ce moment que les théories de Grassé sur la stigmergie s'appliquent sans doute. Progressivement d'autres rayons seront construits parfois dans des positions défectueuses, ne respectant pas le parallélisme (à quoi les Abeilles tendent toujours quand des obstacles insurmontables ne s'y opposent pas). C'est à l'aide de retouches continuelles - phénomène fondamental sur lequel DARCHEN a mis très justement l'accent - que l'ouvre est finalement réalisée ; elle n'atteint nullement d'emblée, comme beaucoup auraient pu le croire, son aspect définitif. Ces retouches peuvent comprendre des destructions plus ou moins massives. On croirait positivement assister parfois à la recherche d'une solution, par une "intelligence " qui tâtonne et cherche à s'exprimer en " termes de cire ". Le rayon en construction présente des zones d'accroissement nettement définies (la partie inférieure de l'arête) et montre des phénomènes de régénération après ablation d'une partie de sa surface. Les régénérations ou l'accroissement peuvent être arrêtés par l'interposition dans l'arête du rayon d'obstacles tels qu'une mince feuille métallique. Si elle est suffisamment large, elle arrête l'accroissement du rayon, à moins qu'on n'y pratique des trous d'un diamètre et à un écartement convenables; alors la construction reprend et englobe peu à peu le métal dans la cire. On voit alors des Abeilles se tenir par une patte à travers les trous; d'autres sont à cheval sur l'arête, qui paraissent tout à fait inactives et se contentent de maintenir leurs pattes accrochées à celles des Abeilles de l'une et l'autre face qu'elles relient entre elles. Eit là où progresse la construction on trouvera tou- 
jours, de la même façon, des masses profondes d'ouvrières qui se tiennent par les pattes. Comme le souligne DARCHeN, on n'échappe pas à la conclusion qu'une information doit passer, très probablement par les pattes ; elle consiste peut-être en de subtiles différences de tension musculaire perçues par les nombreux organes chordotonaux que possèdent ces appendices. Si l'on songe aux innombrables modalités de la construction, il s'agirait alors d'un véritable "langage par modulations tactiles " comparable peut-être aux danses des Abeilles ; ces deux modes d'expression ne sont sans doute pas les seuls, comme certaines expériences et observations réalisées à Bures semblent le montrer. Il en existe probablement un troisième dans les battements antennaires entre ouvrières; et un quatrième sans doute dans les subtils marquages olfactifs au moyen des épagines et des répulsines substances déposées sur les corps que touchent les Abeilles et qui informent tout leur comportement (voir plus loin). Parmi les plus importantes expériences de DARChEN, il convient de citer :

$\mathrm{r}^{\mathrm{o}}$ celles des obstacles interposés sur les tranches des rayons dont il vient d'être question;

$2^{\circ}$ celles sur le parallélisme des rayons et sa régulation, qui sont particulièrement élégants : une lame de cire insérée perpendiculairement entre deux rayons est tordue très rapidement par les Abeilles ; une autre insérée trop près d'une plaque de carton qui recouvre le rayon s'y trouve rapidement collée, sauf si ce carton est percé d'une étroite fenêtre qui permet aux ouvrières de percevoir la surface des cellules du rayon ; la lamelle de cire sera alors non plus collée, mais étirée en respectant le parallélisme. Un des facteurs du parallélisme est donc la cire non pas à l'état brut, mais étirée en cellules;

$3^{0}$ celles sur l'influence de la reine qui favorise ou plutôt détermine la construction probablement par l'intermédiaire d'une hormone analogue à la phérormone;

$4^{\circ}$ plus récemment la démonstration de l'influence régulatrice du fond des cellules, obtenue en transposant des cloisons de cellules de mâles sur des fonds de cellules d'otırières et vice-versa.

Est-il possible de présenter de tout cela une interprétation générale? Pas encore sans doute bien que nous puissions discerner certaines " lignes de force " qui pourraient bien nous y conduire. Il est certain par exemple que le rôle de la grappe des Abeilles cirières est essentiel et qu'il faudrait pouvoir l'observer directement au lieu de procéder, comme l'a fait DARCHEN jusqu'à présent, par inférence, après l'œuvre terminée. Ce n'est qu'en ces toutes dernières années que nous pensons avoir trouvé un moyen d'y parvenir. La construction en effet s'accompagne d'une élévation thermique considérable ; or la masse des Abeilles qui nous dissimule leur travail a en partie pour tâche d'entretenir cette température élevée; si l'expérimentateur y pourvoit de lui-même, ce qui est facile dans un nucléus vitré à un seul rayon, alors la grappe est beaucoup moins dense et il semble qu'on puisse suivre plus aisément le travail des Abeilles. Quant à la difficulté qui gêne tellement tous les chercheurs dans l'étude des insectes sociaux, à savoir où réside la notion de plan du nid, il semble que les travaux de DARCHEN laissent entrevoir un moyen de l'attaquer: si en effet les premières ébauches de la construction peuvent raisonnablement s'expliquer par la notion de stigmergie suivant GRÁsś́, par la suite l'idée d'interconnexion fournirait peut-être une base d'explication plus appropriée aux étonnantes retouches et régulations qui sont monnaie courante dans la ruche. Il semble en effet impossible et absurde que le cerveau d'une Abeille isolée puisse se former une notion précise des 
rayons et des modifications à y apporter. Mais qu'arrive-t-il si les différents cerveaux arrivent à se connecter et si l'essaim parvient à réagir plus ou moins comme un seul animal, somme de toutes les ouvrières? Or, cette connexion, nous la voyons réalisée sous nos yeux au moyen des pattes, dans la grappe cirière...

\section{La CONSTRUCtion Des Cellui,es Royares. Travaux de Vuillaume.}

Mais il est dans les ruches un objet très particulier, les cellules royales, qui focalise l'attention des Abeilles à des moments déterminés de l'année. Vuilfaume a étudié méthodiquement les facteurs de leur construction en présentant aux ouvrières des cupules artificielles de cire et d'autres matières dans lesquelles étaient introduites de très jeunes larves; c'est le principe da l'élevage industriel des reines, familier aux apiculteurs. Il s'est aperçu tout d'abord que les Abeilles acceptaient les cupules de cire, mais aussi celles de paraffine, de verre ou de matières plastiques. I,eur forme a une certaine importance : les cupules carrées par exemple sont moins bien acceptées que res rondes; la taille, l'espacement sur les barrettes porte-cupules, etc. sont à considérer aussi. Par contre la caste et l'âge de la larve ont moins d'importance; par exemple, les larves de mâle peuvent être acceptées, et même dans la nature, il arrive de trouver des nymphes de mâles à l'intérieur des cellules royales. Les cellules sont d'ailleurs l'objet de soins vigilants; la moindre brèche y est promptement réparée comme l'avait signalé d'ailleurs DARCHEN à propos des cellules d'ouvrières. Non seulement en effet les abeilles savent construire, mais encore elles peuvent remédier aux commages très variés que peut causer l'expérimentateur à leurs bâtisses.

Si intéressants que puissent être les résultats de Vuir.aunE en eux-mêmes, il le deviennent plus encore, me semble-t-il, par les lueurs qu'ils jettent sur toute une série de régulations à l'intérieur cle la ruche. Ėn effet, j'ai employé tout à l'heure le terme d'acceptation: il s'agit d'une phase bien distincte de la construction des cellules royales à partir des cupules artificielles. On peut apprécier par le pourcentage de celles qui réussissent les plus oí moins grandes dispositions des Abeilles à réagir ; or les cupules neuves sont souvent refusées, sauf si on les fait séjourner à l'intérieur d'une ruche, même si une toile métallique empêche les Abeilles d'entrer en contact avec elles. Mais le bénéfica de cette familiarisation se trouve perdu si on abandonne ensuite ces cupules cinq ou six jours à l'air libre avant d'y greffer une jeune larve et de les introduire dans la ruche d'élevage. On est donc contraint d'admettre l'existence d'une substance de familiarisation; suivant un terme que j'ai proposé plus tard d'une "épagine " (d'un mot grec qui signifie je place dessus) volatile ou instable, non spécifique d'une ruche déterminée, qui aide les Abeilles à accepter les objets étrangers. VUII.AUME a remarqué aussi que certaines cires provenant de l'extraction de vieux rayons par l'acétone sont régulièrement refusées; elles acceptent volontiers par contre les cires extraites par le benzène. Or la propolis est bien soluble dans l'acétone et non dans le benzène et il est facile de montrer que la "répulsine " est effectivement contenue dans la propolis ; nous verrons plus loin d'autres exemples d'épagines et de répulsines. Nous ne comprenons pas encore très clairement tous les détails de leur rôle physiologique dans l'élevage des reines. VuilzaUme a montré toutefois que la phérormone des reines inhibe l'acceptation des cellules royales quand on en badigeonne les cupules ; d'autre part, des cuptules peuvent être acceptées, même dans une ruche pourvue d'une reine en liberté. Si bien que l'action inhibitrice de la subs- 
tance royale, ou phérormone, n'est pas si radicale que le voulait BUTLER : elle arrêterait seulement la construction spontanée des cellules royales. Mais quand on offre des cupules dont l'élevage a déjà été entrepris par une autre ruche, alors l'acceptation peut se réaliser facilement; seule la phase de début est sensible à l'inhibition de la phérormone.

\section{LES RAPPORTS ENTRE LA REINE ITT I,ES OUVRIỲRES. LA PHÉRORMONE.}

Il y a fort longtemps que l'attraction quasi magnétique exercée par les reines sur les ouvrières a frappé les chercinıurs. On sait depuis Hess que l'influence de la reine ne se borne pas à cette attraction : lorsqu'elle est présente en effet, les ovaires des ouvrières restent atrophiés; lorsqu'on l'enlève, ils grossissent. Hess a constaté aussi que la reine enprisonnée dans une cagette fermée par une seule toile métallique continue à exercer son influence : les ouvrières la nourrissent en passant leur trompe à travers les mailles et leurs ovaires restent petits. Mais si une dottble grille $(5-7$ millimètres de distance entre les deux éléments) empêche l'accès des ouvrières alors leurs ovaires grossissent et il peut même apparaitre des ouvrières pondeuses. Nous sommes donc obligés d'admettre que la nyystérieuse influence ne peut consister dans une odeur de la reine, qui traverserait aussi bien deux toiles nétalliques qu'une seule. Il doit s'agir d'une chimioréccption de contacl au sens de I ÉrTHER, c'est-à-dire d'une perception intermédiaire entre l'olfaction et la gustation et qui paraît très répandue chez les Insectes. C'est sur ces entrefaites que MHe PAN, qui étudiait depuis longtemps ces phénomènes à Bures, fit une curieuse découverte: pour que s'exerce son influence, il n'est pas nécessaire que la reine soit vivante. Une reine morte provoque aussi l'inhibition du développement ovarien che\% les ouvrières, et même s'il s'agit d'un spécimen conservé depuis trois ans dans un carton à insectes! Nous sommes alors contraints d'admettre que l'action de la reine s'effectue par un médiateur purement chimique particulièrement stable puisqu'il peut subsister pendant trois ans. Il importait dès lors d'en connaître la nature. Les purifications commencèrent avec l'aide de M. BARbir du I aboratoire de Chimie biologique de la Sorbonne à partir de plusieurs kilos de reines que nous nous procurâmes par divers moyens. Après plusieurs années d'efforts, Mlle PAIx se trouva en mesure de distinguer plusieurs substances qu'on peut désigner sur la proposition de BUTExANDT et KARIsOx sous le nom de phérormones. Io) une phérormone I isolée par BuTrı́n, et en même temps par BARBIER et LEDERER : c'est 1'acide céto-g décène-2-transoïque. $2^{\circ}$ ) une phérormone II, qui comprend deux ou peut-être trois substances. I a phérormone I n'a aucune propriété attractive et n'inhibe pas les ovaires si on la mélange à la nourriture. C'est le mélange phérormone $I+I I$ qui estattractif ct inhibeles ovaires. MHe PAIN pense d'ailleurs que les autres effets de la phérormone (tels que ceux sur la construction, étudiés par DARCHEN) pourraient servir éventuellement à mettre en évidence d'autres constituants du complexe de la phérormone.

Le développement des ovaires ne dépend pas uniquement de facteurs négatifs, mais aussi de facteurs positifs tels que la qualité de la nourriture, et essentiellement des protéines qui sont apportées par le pollen. A ces protéines s'ajoutent vraisemblablement une série de vitamines du groupe B ; il est probable aussi que les modifications que subit le pollen entreposé à l'intérieur de la ruche ne sont pas sans importance. Notons aussi la présence d'un effet de groupe à déterminisme complexe : 
les ovaires des Abeilles isolées se développent avec un grand retard et l'échange social de nourriture paraît indispensable au développement de ces organes; mais il est extrêmement curieux de noter que dans les groupes de deux Abeilles, l'une a presque toujours des ovaires plus gros que l'autre (la différence diminue par la suite) comme si l'une agissait plutôt comme donneuse et l'autre comme distributrice de nourriture, Enfin des périodes critiques étroitement liées à l'âge des jeunes Abeilles, ontétéégalement mises en évidence par Mlle PAIN ; au-delà ou en deçà de ces périodes, 1'administration de nourriture azotée n'a que peu ou pas d'effet sur le développement ovarien.

\section{LES MĹCANISMES DE DÉFFNSE DE LA COIONIE.}

Évidemment, les colonies d'Abeille, qui sont très "fermées " au sens de LE MASNÉ, possèdent pour la défense du nid toute une série de coordinations sociales. LECOMTE en a fait l'objet de ses travaux. Il a remarqué d'abord qu'en cagettes et à l'étuve, les Abeilles n'attaquaient que les objets en mouvement, surtout s'ils sont de teinte foncée, pileux, et sentant le venin d'Abeilles. Da plus, ce sont les ouvrières âgées qui sont spécialement agressives, surtout si leurs ovaires sont bien développés. Deux groupes. d'Abeilles ne s'attaquent pas si elles ont pu échanger de la nourriture; par contre deux groupes prélevés dans la même ruche au même moment se battront férocement si on les réunit après une séparation de trois ou quatre jours. Ce n'est point l'odeur combinée des fleurs qui ont fourni le nectar (combinaison qui caractérise chaque ruche) qui est à la base de cette différenciation, comme le soutient RiBBANDs : mais une élaboration particulière qu'en fait chaque groupe d'Abeilles. Dans la nature, les phénomènes sont un peu différents; d'abord, l'attaque ne s'opère qu'avec un leurre de grande taille, bien supérieure à celle d'une abeille. D'autre part, les butineuses suivent dans l'air des voies déterminées, le long desquelles elles se montrent très agressives ; elles manifestent de plus une certaine " territorialité " et on peut mettre parfois en évidence des comportements de défense de l'aire de butinage. C'est là que les recherches de LECOnIE ouvrent à mon sens une voie curieuse et originale vers une meilleure compréhension des procédés de marquage des Abeilles. J'ai cherché à les synthétiser en groupant les résultats de DARChEN, VuIL, AUME, M11e PAIN et LacomTE. Sur les billes de verre qu'on dépose dans un nourrisseur pour éviter qu'elles ne se noient, les abeilles déposent un mélange complexe de substances, une "épagine ". (voir plus haut) spécifique de la ruche qui n'attire que les ouvrières de cette ruche ; elle est mélangée d'une " répulsine " qui n'exerce son activité " de dissuasion " que vis-à-vis des ouvrières d'une autre colonie. Or il existe, nous l'avons vu, bien d'autres. substances dans la ruche qui agissent comme épagines ou comme répulsines. I1 semble que le comportement de l'Abeille soit souvent guidé comme par des rails à 1'aide de ces touches chimiques, qui l'avertissent de s'approcher ou de s'éloigner de tel ou tel objet.

\section{UNE RÉGULATION SOCIALE INATTENDUE, L'ANTISEPSIE SOCIALE}

En I950-5I, mon collaborateur IAVIE s'occupait d'étudier une intéressante levure Kloekera apiculata apis qui vit dans les troncs trachéens des abeilles et dont il avait pu mettre en évidence l'action toxique vis-à-vis de l'Acarapis woodi, agent d'une très grave maladie, l'acariose des Abeilles. Des essais de culture, puis d'infes- 
tation artificielle avec des Kloeckera de ruches acariosées avaient bien réussi. Je lui conseillai donc d'étudier la propagation de cette levure dans la ruche ; j'aurais aimé savoir en particulier comment les Kloeckera parvenaient à pénétrer dans les trachées. Donc, après avoir pulvérisé dans la ruche une suspension de ces levures, LaviE frottait la fourrure des ouvrières d'un fil de platine stérile et essayait par culture d'y retrouver les levures, sans beaucoup de succès d'ailleurs. Mais après avoir ensemencé ainsi quelques centaines de tubes, il me fit part de son étonnement : presque rien ne poussait dans ces tubes, ni bactéries, ni levures, ni champignons. Je restai d'abord très sceptique ; comment croire que la fourrure de l'Abeille, qui entre en contact avec une foule de végétaux, ne collecte pas aussi une infinité de poussières et de spores? Je conseillai done d'abord de recommencer et d'augmenter le nombre des cultures, et de poursuivre des essais parallèles avec les mouches qui se promenaient sur les vitres du laboratoire. Après quelques jours, il fallut bien se rendre à l'évidence : les ensemencements à partir des Abeilles ne donnaient toujours presque rien. A partir des mouches au contraire, une flore dense et variée envahissait les tubes. Il n'y avait guère qu'une conclusion possible: une substance antibiotique devait détruire les germes que les Abeilles ne peuvent manquer de transporter. C'est alors que nous nous rappelâmes une très ancienne observation de WHITE, au début du siècle, et qui n'attira aucune attention à l'époque; cet auteur avait eu l'idée d'étudier la flore interne de la ruche et avait frotté le fil de platine stérile contre les rayons ; il fut très surpris de sa maigre récolte, du point de vue qualitatif et quantitatif ; il semblait que les ruches soient très pauvres en microorganismes associés. Ne s'agirait-il pas là aussi d'un antibiotique? Notons d'ailleurs que les propriétés antibiotiques de la propolis et de la gelée royale sont bien connues et nous avons montré à Bures même la présence d'une substance antibactérienne dans différents pollens. Ne peut-on généraliser? N'existerait-il pas dans la ruche tout un ensemble de substances antibactériennes qui empêchent la prolifération des germes dans un milieu où circulent tant de poussières et matières fermentescibles? C'est la question que LAviE se posa; et il réussit à extraire et à doser biologiquement des substances antibactériennes dans le corps des Abeilles, la propolis, la gelée royale, le pollen, la cire et le miel. C'est justement cette défense proprement sociale contre les infections (car l'Abeille isolée ne participe à la récolte d'aucune de ces substances) qui explique la pauvreté de la flore, signalée par WHITE.

\section{I'ABEILLE ET IAA RÉCOLTE DU POILLN. TRAVAUX DE I,OUVEAUX.}

Au cours de recherches minutieuses qui ont duré un grand nombre d'années, I.OUVEAUX a déconvert plusieurs caractéristiques importantes et nouvelles de la récolte du pollen. D'abord, les récoltes sont qualitativement et quantitativement très hétérogènes ; il n'existe sans doute pas deux ruches, même placées côte à côte, qui aillent visiter les mêmes fleurs dans les mêmes proportions. Le facteur le plus important qui régisse l'activité de récolte, c'est la ponte de la reine et la nécessité de fournir au couvain l'azote dont il a besoin pour sa croissance. Mais justement, pensa Louveaux, puisque le pollen est la seule source d'azote de l'Abeille, comment varie la répartition de cet élément dans la récolte? C'est à quoi personne n'avait pensé jusque-là. Les dosages exécutés par LoUvEAux d'une façon systématique permirent alors d'apercevoir un dénominateur commun caché sous les diversités botaniques. 
D'abord, il existe un cycle de l'azote du pollen au cours de l'année; les pollens les plus riches sont ceux du printemps et du début de l'été ; leur taux moyen en azote baisse ensuite sensiblement. Ensuite, certaines ruches manifestent des caractères très stables : elles sont bonnes ou mauvaises récolteuses d'azote, ce qui influence évidemment leur développement. Enfin et surtout, les ruches d'une région transplantées dans une autre conservent pendant une année au moins des particularités de récolte qui permettent de les distinguer des ruches indigènes: les pollens récoltés ne sont pas les mêmes, dans les même proportions, dans ces groupes transplantés, que dans les groupes indigènes. Comme Louveaux l'a montré à la suite de mesures très ardues, c'est encore et toujours leur cycle propre de récolte de l'azote implanté dans leur patrimoine héréditaire (et probablement d'abord dans le cycle de ponte de la reine) qui régit le choix qu'elles feront de pollens plus ou moins riches en cet élément. Ces conclusions très importantes expliquent les difficultés d'acclimatation connues depuis longtemps des apiculteurs.

\section{AUTRES RECHERCHES.}

Cette synthèse, très brève, encore une fois, ne porte que sur les travaux les plus suivis et dont les développements ont été les plus importants. Mais il va de soi qu'au cours des années, nous avons dû jeter des coups de sonde dans bien d'autres directions. J'ai étudié moi-même comme je l'ai dit l'effet de groupe c'est-à-dire la mortalité moyenne en fonction du groupement; les ouvrières isolées meurent en effet très rapidement, mais subsistent normalement lorsque le groupe est suffisamment dense ; sans doute l'échange mutuel de vitanines y est-il pour quelque chose. VuILIAUME a enregistré les mouvements respiratoires des Abeilles, et LE BIGOT a mis en évidence une teneur en gaz carbonique très élevéa dans l'atmosphère des ruches, ce qui a été retrouvé par les Russes dans des conditions un peu différentes. Il a aussi, au cours d'études sur la claustration des ruches, montré que l'apport d'eau en grande quantité permet d'éviter la mortalité subséquente ; c'est d'ailleurs une question en plein développement, et il est probable que nous pourrons d'ici peu entretenir les lecteurs des Annales de l'Abeille de l'approvisionnement artificiel en eau et de ses conséquences. VurL.LAUME a montré aussi la persistance des impressions mémoniques qui paraissent ne s'effacer qu'avec la vie chez la butineuse, contrairement à ce que l'on pensait. LAVIE a étudié le comportement calorifique des ruches vides de différentes constructions, ainsi que le thermopreferendum des Abeilles; il a vu avec RoTH, que le facteur social l'emportait de beaucoup sur tous les autres, ce qui m'a inspiré l'idée de faire étudier de la même façon l'hygropreferendum et le photopreferendum (par VERRON et ROPARTZ); ces chercheurs ont abouti à des conclusions voisines : à rien ne sert d'étudier ces types de comportement chez l'Abeille isolée, pour laquelle toutes les réactions sont pathologiques ; et d'autre part lorsqu'on les mesure dans le groupe d'Abeilles, on voit apparaitre une sorte d'isostasie sociale qui prive la recherche de son sens. Gorliot qui nous a quitté maintenant, a construit un hygromètre sonde, le plus fidèle qui soit sans doute parmi les appareils du même principe; et il a fractionné la fumée qui sert à calmer les abeilles pour en découvrir le principe actif ; c'est sans doute un mélange d'acide acétique, d'acétone et d'alcool méthylique. Louveaux a beaucoup étudié la technologie du miel, le mode de chauffage qui convenait le mieux pour le fondre, le comportement des grains de pollen dans le miel, etc. Ces questions 
tendent à constituer une part de plus en plus grande de son activité. LECOMTE a mis en évidence un facteur olfactif et un vibratoire dans l'interattraction si nette qui amène les Abeilles à former une grappe serrée; et il s'est attaqué, avec CorABOEUF et BOISTEL, à l'électrophysiologie des récepteurs antennaires. DARCHEN et VUILLAUME enfin ont découvert deux types de cellules royales dans la ruche, suivant les circonstances dans lesquelles est commencé l'élevage royal; il existe sans doute d'après ces mêmes auteurs, des "pré-reines ", larves qui reçoivent une alimentation plus riche en gelée et peuvent donner plus tardivement des reines ou tout au moins des formes intermédiaires. DARCHEN enfin a repris d'anciens travaux allemands sur la polygynie expérimentale, obtenue en sectionnant l'aiguillon des reines qui ne peuvent plus alors se massacrer mutuellement; mais ces études n'en sont encore qu'à leur début.

\section{CONCLUSIONS}

Au terme de cette synthèse des travaux de treize ans, il me semble qu'on peut en dégager une certaine orientation générale : nous nous sommes surtout intéressés à ce qui est proprement social dans la vie de la ruche; ce sont surtout les mécanismes internes dans la vie de la colonie qui ont retenu notre attention, à l'exclusion de la danse des Abeilles. Le champ à explorer était vaste et il le reste. Pour l'avenir nous comptons progresser dans la même direction en nous localisant sur quelques points plus précis :

I) problème des substances actives, épagines, répulsines, allectines (ces dernières attirent les Abeilles vers le miel, le pollen et pent-être la propolis) hormones sociales (dont il existe sûrement d'autres que celles de la reine);

$\left.2^{\circ}\right)$ problème des échanges de nourriture en égard au développement de la colonie et peut-être de l'ovaire de la reine; et aussi facteurs du développement des mâles que l'on a trop négligés jusqu'ici;

$3^{\circ}$ modes d'expression et de transmission socianx employés au cours de la construction, battements d'antennes, etc.

$4^{\circ}$ transmission des "traditions de récolte " au cours du si curieux phénomène de l'acclimatation.

\section{LISTE DES TRAVAUX DU LABORATOIRE DE BURES-SUR-YVE'T'TE :}

\section{$1949-1950$}

(i) Chauvin R., i950. La question de la sélection et de l'amélioration des races d'Abeille. Rev. Zool. Agr. Appl., I-I I.

(2) Chauvin R., i950. Méthode d'observation continue de la ponte de la reine chez les Abeilles. L'Apicul. teur, (sect. sc.), $57-68$.

(3) Chauvin R., I950. Sur l'insémination artificielle des Reines d'Abeille sans appareils compliqués. L'Apiculteur, $69-74$.

(4) Derromont et al. I950. Sur l'excrétion de quelques colorants chez l'Abeille. Arch. Intem. Physiol, $58,343-9$.

(5) LAviE P., I950. Nouvelles recherches sur le traitement de l'Acariose. Ren. Fr. Apicult., 21-24.

(6) Lavie P., i950. Sur un organisme énigmatique (Acaromyces Laniae) qui semble s'attaquer à l'Acarapis Woodi. L'Apiculleur, 4I-45.

(7) Lecomte J., I949. L'interattraction chez l'Abeille. C. R. Acad. Sci, 229, 857-858. 
(8) Leconte J., I950. Attraction entre reine et ouvrieres (.I pis mellifict). C. R. Acal. Sici, 231, 8o2-Sot. (9) LecomTe J., I950. Sur le déterminisme de la formation de la grappe chez les Abeilles. \%. vergl. Physiol., $32,499-506$.

(10) Louviex J., ro +9 . Litudes sur la toxicite et le pouvoir répulsif vis-à-vis de l'Abeille de divers insectirides. Ren. Fr. Apicull, 1-1 2.

(11) Louveaux J. ét al., 1950. Études concemant la définition de l'état de floraison de certaines Curucifères oléagineuses dans la région parisienne. L'Apiculeur, 9-3t.

(r 2) LouveatX J., 1950. Observations sur le déterminisme de la récolte du pollen par les colonies d'Abeilles. (Apis nellifica L.) C. R. Acad. Sci, 231, 92 r-922.

(13) LoUveaUX J., I950. Utilisation d'un test biologique pour la caractérisation de certaines intoxications d'Abeilles par des produits antiparasitaires. L'Apiculleur, 35-10.

(14) Pain J., 1950. Contribution à l'étude cle l'ovairc des ouvrières d'Abeilles. L'Apiculteur, 45.55.

(15) Vund Aume M. el al., Étude des mouvements respiratoires de l'Abeille au moyen de l'enregistrement optique. Arch. intern. Physiol., 57, 383-392.

1951

(a6) Cinavix R., 195I. Sur le déteminisme de leffet de groupe chez les Abeilles. Physiol. Comp. Uecol, $282-288$.

(17) Chatvin R. el al., r951. Voyage d'étude apicole en Allemagne. Rev. Fr. Apicull., 6o8-6or).

(1.s) Cinuvin R., 1951. Sur les altérations du miel. L'Apiculleur, zi-26.

(ig) Chauvix R., r95I. Valeur thérapeutique du miel. L'. Ipicultew, 29-39.

(20) DARcien K., 195r. Sur quelques régulations sociales dans la construction chez les Abeilles. $C$. $R$. .tad. Sci, 234, 67 - 3 .

(21) Lavis P., 1951. Essai d'étude themique de la ruche non peuplée. L'A picullestr, 1-12.

(22) LECONTE J. Recherches sur le comportement agressif des ouvrières d'Apis mellifica I. Beluaioui, $60-66$.

(2.3) Lecomte J., з95 . Les facteurs de l'agressivité chez l'Abeille. C. R. Acad. Sci., 232, 1.376-8.

(24) PaIn J., L'alimentation et le développement des ovaires chez l'ouvrière d'Abeille. Arch. intern. Physiol., 59, 203-210.

(25) Pain J. i95I. Vitamines et développement ovarien chez l'ouvrière d'Abeille. C. R. Soc. Biol., 165, $1505-7$.

(26) Vercé J., r95. L’activité antibactérienne de la propolis, du miel et de la gelée rovale. L'A ficulteur, I $3-20$.

\section{2}

(27) Cinuvin R., 1952. Déterminisme du polymorphisme social chez les Abeilles. C.N.k.S. Symp. Soc. An., II 7 -I 22.

(28) Chauvin R., 1952. Xouvelle technique d'enregristrement de l'activité de la ruche. L’Apicalleur, 9-I4.

(29) Chalvis R. et al. Sur une substance présente dans le pollen qui s'oppose au développement de certaines bactéries. C. R. Soc. Biol., 166, 645.

(30) Cuauvin R., 1952. Sur la claustration des ruches. L'Apiculteur, 27-32.

(31) Darcien R., I952. Regulation sociale et écartement des rayons chez les Abeilles. C. R. Acad. Sci., $235,820-822$.

(32) Golloror $\mathrm{C}$., 1952. Un nouvel hygromètre spécialement conçu pour l'étude des microclimats au laboratoire ou à l'extérieur sous abri. Arch. Meteo. Geoph. Bioklim., 4, 207-2I 2.

(33) Lavie 1', 1952. Premiers résultats obtenus dans les essais de traitement de l'Acariose au moyen de 1'. Acaromyces. L'A piculteur, I-8.

(34) Lecomte J., 1952. IIétérogénéité dans le comportement agressif des ouvrières d'Apis mellifica. $C R$. Acad. Sci., 234, 89o-89т.

(35) Louviaux J. et al., 1952. Recherches sur la pollinisation chez la Navette (Brassica rapa, var. oleifera). L'Apiculteur, I5-18.

(36) Verce J., I952. Sur un microorganisme du genre Serratia, agent pathogène des larves d'Hyménoptères Vespides (Incidences apicoles). I'A Apictlleur, $2 \mathbf{I}-26$.

\section{3}

(37) Chauvin R., I953. Le maintien de la ruche en milieu confiné est-il possible? L'Apiculteur, 25-29.

(38) Le Bigot L., I953. L.es particularités de l'atmosphère interne des ruches. L'Apiculteur, 39-46.

(39) Darcien R., 1953. La régulation sociale et l'écartement des rayons chez les Abeilles. $C$. $R$. Acad. Sci., 237, $1032-4$.

(40) Defromont C., r953. Effets toxiques prolongés sur les Abeilles des traitements sur Colza par esters phosphoriques. C. R. Acad. Agric., Io.

(4I) LAviE P. L'enregistrement thermique continu dans les populations d'Apis mellifica au cours de l'hivernage. Ins. Soc., 1, 39-48.

(4) Lavie P. el al., t953. Sur le themopreferendum et la production de chaleur chez les Abeilles. Physiol. comp. Oecol., 3, 57-62. 
(43) Leconte J., 1953. Relation entre lauge et l'asressivite chez les abeilles ouvrières. L'Apiculteur, 33-38.

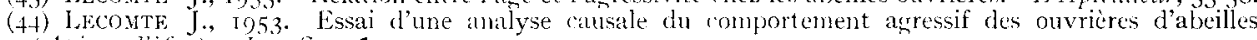
(. lpis mellifica). Ins. Soc., 1, to-57.

(45) Pons J., 1953. La "substance de fécondite " dan le developpement des ovares des ouvrieres d'abeilles (.this mellifica L.). (ritique des travaux de Nüssbichler. Ins. Sor., 1, 6o-7o.

(46) Vergí J., r953. Sur 34 diméthyl 5 sulfanilamidoixoxasol (Gantrisinc) comme agent thérapeutique

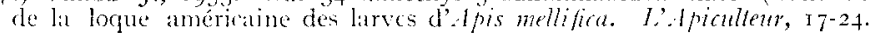

(47) Conuvin R., I954. Asperts sociaux res grande's fonctions chez l'Abeille. La thérie du superorganisne. Ins. Soc., 1, 1 $23-120$.

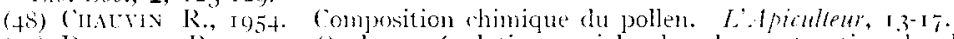

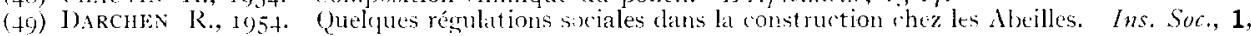
2I (1)-228.

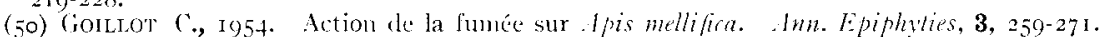

(5I) JAVIE I', 195. Essai de lutte biolnique contre l'Acarapis wooli, anent de l'Acariose de l'Abeille. C. R. Acal. Sci., 238, 947-9+s.

(52) Louvenux J. t al., 1954 . Communiqué sur l'activité de la Commission de Botanique apicole de l'l'. I. S. B. en 1953-1954. L'fipicullent, 1-6.

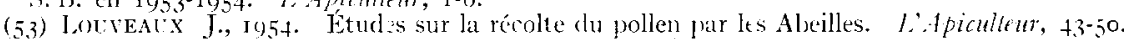

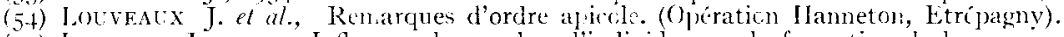

(55) I. .ecomte I., 195." Influnce du nombre d'i dividus sur la formation de la grapje par les ouvrieres "Abeilies. C. R. Acal. Sci., 259, т870-1872.

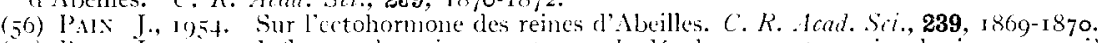

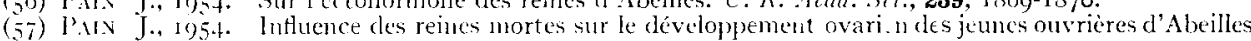
(Ipis mellifica L.). Ins. Sor., 2, 35-43.

(58) Vrokos II., 1954. Ies réations des Abeilles à l'humidité. Ins. Soc., 2, 57-62.

\section{5}

(5)) Cintvin R., I955. La gelée royale: I : Récolte et conservation. Propriétés physiques. L'.lpiculleur, 7-I 2 .

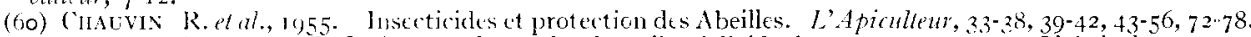

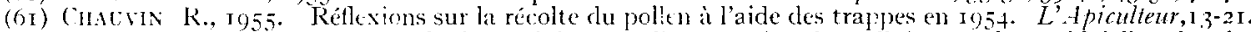

(62) cuncvin R. el al., I955. Sur le déteminisme de lacreptation des cellules royales artificielles rhez les Abilles. C. R. Aat. Sa, 241., I 880 -rogr.

(6.3) Darcien R., ig5.5. Nouvelles régulations clans les constructions d'dpis mellifica. C. R. Acad. Sci., $241, \quad 1081-1083$.

(64) Iavie P., 195. I.enregistrement themique continu dans les populations d'Apis mellifica. Ins. Soc., 2, 127-134.

(65) Louveaux J., ro5.5. Introluction a l'étude de la scolte du pollen par les Abeilles (Apis mellifica L.) Plinsiol. Comp. Oecol., 4, I-5.1.

(66) P’an J., r 955. Dowage biologique et spectrographie de lectohomone des Reines d'Abeillos. C.R. Acad. Sci., 240, $670-672$.

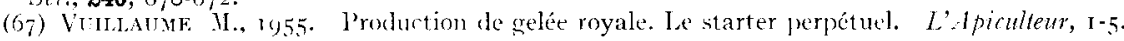

\section{6}

(68) Cnavvin R., Io56. Les farteurs de ponte chez la reine d'Abeilles. Ins. Soc, 3, 499-504.

(69) ('indunin R., 1956. Sur un principe de la creléc royale d'Abeilles, act if sur la glycémie des Mammiferes. C. R. Acad. Sci., 243, 1920-1921.

(70) Cmavis R., 1956. Composition biochimique de la relée royale. L'Apicullure, 45-55.

(7) Cinuvin R. é al., ig56. Étude macroscopique el microscopique de la gelée royale. L'Apicalleur, $3.3-35$.

(72) Cincvin R. at al., 1956 . Les caractures de la récolte du pollen en 1955 . La flore française product rice de pollen pour les Abeilles. L'Apiculleur, iz-28.

(73) Cinuvin R. el al., 1956. Recherrhes sur la substance antibiotique du pollen. Ann. Inst. Pasteur, 90,523 .

(71) BoIster, J. el al. Onelques asperts de l'étude électrophysiologique des récepteurs sensoriels des antemes d'hyménoptères. '(Faculté des siences de Paris).

(75) Cinuvin R. el al., 1956. Action de la gelée royale d'Abeille injectée aux mammiféres. Biologica latina, $9,442-452$.

(76) Cunuvin R. et al., 1956. Le développenent des ovaires des ouvrières des Abeilles et l'ectohormone des reines. Experientia, 12, 354 .

(77) Darchen R., 1956. La construction sociale thez Apis mellifica. Ins. Soc, 3, 29,3-30r.

Annales de l'Abeille. - ig6r. 
(78) GoIllot C. et al, 1956. Études sur la sédimentation pollinique dans les miels fluides au repos. Grana Palynologica, (N.S.), 1, go-98.

(79) GoIllot C., 1956. Recherches de caractéristiques physiques de la gelée royale (G. R.) totale. L'Apiculteur, I-I 6 .

(80) Lecomte J., 1956. Formation de pistes par les butineuses d'une ruche ayant subi une rotation de I80\%. Z. Bienenforschung, 3.

(8I) Lecomte J., I956. Sur le vol des Abeilles butineuses. Z. Tierpsychol., 13, 26-30.

(82) Lecomte J., 1956. Nouvelles reche rches sur l'interattraction chez A pis mellifica L. I95-I 98.

(83) Louveaux J., I956. Remarques sur les facteurs conditionnant le choix par les Abeilles (Apis mellifica L.) des plantes leur fournissant du pollen. C. R. Acad. Sci., 242, 2994-2996.

(84) Louveaux J., I956. Applications des méthodes de l'analyse pollinique à des produits au miel. $L$ 'Apiculteur, $29^{-2} \mathbf{I}$.

(85) Pain J., 1956. Mesure du pouvoir inhibiteur etde l'attractivité de l'ectohormone des reines d'Abeilles. Différences individuelles. C.R. Acad. Sci., 242, 1080-1082.

(86) Vuillaume M, r956. Les stimuli qui régissent l'acceptation des larves introduites dans les cellules royales artificielles chez les Abeilles. Les facteurs dépendant des larves. $C$. R. Acad. Sci., 242, 562-564.

(87) Vuildaume M., 1956. Les stimuli qui réagissant l'acceptation des cellules royales chez les Abeilles : les facteurs dépendant des cellules. C. R. Acad. Sci., 242, 185-IS7.

\section{$195 \%$}

(88) Chauvin R., 1957. La gelée royale : action sur les mamnifères et sur l'Honmc. L'Apiculterm, 89-95.

(89) Chauvin R., I957. Sur les effets physiologiques et thérapeutiques de divers extraits de pollens. Rẽ̃. Path. Gen. Physiol. Clin, 687, 6r I-623.

(90) Chauvin R., et al., i957. Composition et propriétés du pollen récolté par les Abeilles. B. Acad. Nat. Med., 35 .

(9i) Chauvin R., 1957. Sur les propriétés physiologiques de certains pollens et spécialement sur un principe hyperglycémiant. C. R. Acad. Sci., 244, I20-1 22 .

(92) Darchen R. La reine d'Apis mellifica et les constuctions cirières. C. R. Acad. Sci., 439-44I.

(93) DARChen R., I957. Le travail de construction de l'Abeille est-il un travail sccial? L'Apiculleur, IOQ-I 2.

(94) Darchen R. et al. I957. Sur le déterminisme de la construction des cellules de mâles chez les Abeilles. C. R. Acad. Sci., 244, 391-394.

(95) Darchen R., I957. La reine d'Apis mellifica, les ourrières pondeuses et les constructions cirières. Ins. Soc., 4.

(96) Goillot C., I957. Étude physique de la conservation de la grelée royale brute. C. R. Acad. Sci., 245, 1082-1084.

(97) Lavie P., I957. Étude des substances antibiotiques présentes chez Apis nellifica et chez quelques Insectes sociaux. C. R. Acad. Sci., 244, 2653-2655.

(98) Maurizio A. el al, 1957. Rapport d'activité de la Commission Internationale de Botanique Apicole de l'U. I. S. B. pour les années I955-1956. L'Apiculleut, I-6.

(99) Louveaux J., 1957. Botanique apicole. L'A picalteur, 1-6.

(100) Lecomte J., I957. Sur le marquage olfactif des sources de nourriture par les Abcilles butineuses. C. R. Acad. Sci., 245, 2385.2387.

(IOI) Vullaume M., r957. Contribution à la psychophysiologie de l'élevage des reines chez les Abeilles. Ins. Soc., 4, I I-156.

(io2) Vuillaume M., 1957. L'importance de l'approvisionnement en eau dans la ruche. Ins. Soc;, 4, $3 \mathrm{I}-4 \mathrm{I}$.

(ro3) Vululaune M., I957. La forme des cellules royales chez les Abeilles. Ins. Soc, 4, 385-390.

(I04) Vunlaume M., I957. Élevage de Reines. Production de Gelée Royale. L'Apiculteur, Ir3-rí.

(105) Chauvin R., 1958. Les travaux de la Station de Recherches Apicoles de Bures-sur-Yvette en I957. Ann. Abeille, 1, 107-III.

(io6) Chauvin R., 1958. Biologie de l'Abeille. Revue générale jusqu'en 1956. Ann. Abeille, 1, 4I-67.

(IO7) Chauvin R., et al., I958. Le vieillissement du pollen emmagasiné par les abeilles et de la gelée royale. C. R. Acad. Sci., 247, 2020-2042.

(108) Darchen R., I958. Les constructions sociales chez Apis mellifica. Behaviour, (including insect), 529-538.

(IOg) DARChEN R., I958. Les Abeilles peuvent tordre une lame de cire pour la rendre parallèle aux rayons. C. R. Acad. Sci., 24\%, 2208-2210.

(I Io) DaRChen R. et al., Étude préliminaire de quelques qualités de cires gaufrées présentées aux Abeilles. Ann. Abeille, 1, II-I8.

(III) Darcien R. et al., x958. Déterminisme de la construction des cellules de mâles et des cellules d'ouvrières chez Apis mellifica. Ann. Abeille, 1, 7-9.

(II2) LECOMTE J., I958. Que savons-nous du comportement des faux-bourdons? Ann. Abeille, 1, 3I-39.

(i 3) Courtois G. et al., I958. Sur un procédé de marquage des Abeilles butineuses au moyen d'un radioisotope. C. R. Acad. Sci., 247, I 47-149. 
(II4) BoRneck R. el al., I958. Une enquête sur le marché du miel à Paris pendant l'hiver i957-58. Ann. Abeilles, 1, 223-246.

(i 15) Louveaux J. el al., 1958. Étude technique sur la fonte du miel cristallisé. Ann. Abeille, 1, 19-30.

(i i6) Louveaux J., I958. Recherches sur la récolte du pollen par les Abeilles (Apis mellifica). (Thèse Paris). (I 7) Vuillaume M., I958. Technique d'élevage des reines. I. - Le premier stade : élevage des cellules royàles ; étude critique. Ann. Abeille, 1, г89-196.

( I I8) Vuillaume M., I958. Essai de modification des réactions instinctives des Abeilles au cours de l'élevage royal. C. R. Acad. Sci., 246, 2 I69-2 I 7 I.

( I 19$)$ Vurltaume M., i 95\%. Les substances inhibitrices de la construction des cellules royales chez lesAbeilles. C. R. Acad. Sci., 246, 1 $298-1299$.

(г 20) Vulllaume M., ig58. La théorie de Butler sur l'ectohormone des Abeilles et ses limites. C. R. Acad. Sci., 246, 1927-1929.

\section{9}

(12 I) Cilauvin R., 1959. Travaux de la Station de Recherches apicoles de Bures-sur-Yvette en I958. Ann. Abeille, 2, I85-I90.

(i 22) Ciracvin R., I959. Action de la gelée royale sur les animaux supérieurs et sur l'Homme. Critique de la revue des Johansson. Ann. Abeille, 3, 223-234.

(123) Chatvin R., i959. La valeur diététique et thérapeutique des produits de la ruche. Miel. Pollen. Gelée royale. Produits pharm., 14, I-27.

(1 24) Cotrtors ( ., et al., I959. Sur la résistance au rayonnement gamma de l'Abeille ouvrière. Ann. Abeille, 2, 285-290.

(i25) Cocr'Tols G. et al., 1959. Marquage d'Abeilles au moyen d'or radioactif. Intern. J. Appl. Rad. Isotopes, 5, 265-269.

(126) DARCHEN R., 1959. Les techniques de constructions chez Apis mellifica. (Thèse. Paris).

(I27) DARCIEN R., I959. Observation et expérimentation sur un essaim nidifiant artificiellement à l'air libre. Ann. Abeille, 2, 5-12.

(I 28) Darchen R., I959. Un des rôle des chaînes d'Abeilles : la torsion des rayons pour les rendre parallèles entre eux. Amn. Abeille, 2, I93-209.

(i 29) Darchen R., I959. Construction et reconstruction de la cellule des rayons d'Apis mellifica. Ins. Soc., 4, $357-37 \mathrm{I}$.

(I 30) DARCHen R., I959. Revue critique. L'Abeille, insecte social. J. Psychol., 75-97.

(131) DARchen R. el al., r959. Deux types de cellules " royales "chez Apis mellifica. Existe-il chez Apis mellifica des sexués de remplacement?. Ann. Abeille, 2, 177-183.

(I32) Freskaye J., I959. Note technique. Protection du bois des ruches. Le paraffinage. Ann. Abeille, $2,235-256$.

(I 33) LAvis P., 1959. Action antibiotique de quelques substances provenant de l'Abeille ou de la ruche sur Bacillus lanae et sur Bacillas alvei. C. R. Acad. Sci., 248, 455-457.

(I34) Lavie P'. el al., 1959. Les rapports entre la substance antibiotique des reines et des ouvrièresd'Abeilles, le développement ovarien et l'ectohormone. C. R. Acad. Sci., 248, r 587 - 589 .

(I35) LAVIE P. el al., I959. Relation entre la substance attractive, le facteur antibiotique et le développement ovarien chez la reine d'Abeille, Apis mellifica. C. R. Acad. Sci., 248, 3753-3755.

(136) LeConte J., 1959. Luzerne et apiculture. Ann. Abeille, 2, 211-22I.

(I37) LeComTE J., I959. Premières observations sur le comportement des insectes pollinisateurs de la luzerne Ann. Abeille, 2, $277 \cdot 284$.

(138) Leconte J. el al., 1959. Non toxicité pour les Abeilles des traitements à base de Bacillus Thuringiensis, souche anduze. Ann. Abeille, 2, 17 1-175.

(139) Louvfaux J., r9 59. Technologie du miel. Ann. Abeille, 2, 343-354.

(i40) PAIN J., I959. Étude de l'apparition de l'attractivité chez les reines vierges d'Abeille. C.R. Acad. Sci., 248, $32 \mathrm{II}-32 \mathrm{I} 2$.

(I4I) VullLAUme Y., I959. Nouvelles données sur la psychophysiologie de l'élevage des reines chez Apis mellifica. Ann. Abeille, 2, I $13^{-1} 3^{8}$.

(142) Vuillaume M., I959. La rétention mnémonique chez Apis mellifica. Ann. Abeille, 2, I59-I70.

(i43) Vuiliaume M., r959. Les productions simultanées de miel, gelée royale et Abeilles, sont-elles incompatibles? Ann. Abeille, 2, 271-275.

\section{0}

(144) Chauvin R., 1960. Travaux de la Station de Recherches Apicoles de Bures-sur-Yvette en I959. Ann. Abeille, 3, 307-3r3.

(I45) BARBIER M. et al., I960. Étude de la sécrétion des glandes mandibulaires des reines et des ouvrières d'Abeilles (Apis mellifica) par chromatographie en phase gazeuse. C. R. Acad. Sci., 250, 3740-3742.

(i46) Chavvin R. I960. Progrès récents dans la biologie de l'Abeille. Ann. Abeille, 3, 5-39.

(147) Chauvin R., rg60. Les substances actives sur le comportement à l'intérieur de la ruche. Ann. Abeille, 3, $185^{-197}$.

(I48) DARCHEN R., I960. Die Bienengemeinschaft. Biene und Bienenzucht, 53-67.

(i49) Darchen R., I960. L'ablation du dard des reines et des ouvrieres d'Apis mellifica. Applications pratiques. C. R. Acad. Sci., 250, 203-204. 
(150) Darchen R., ig6o. Le polygynisme expérimental dans les sociétés d'A pis mellifica. C. R. Acad. Sci., 250, $934-93^{6}$.

( 5 I) DARCHEN R., I960. Les régulations neurohormonales de l'instinct constructeur des ouvrières d'Apis mellifica. Compléments aux observations de Roscir et de Moskovijevic. Ann. Abeille, 3, 329-333.

(152) Fresnaye J., 1960. Deux années expérimentales sur la protection du bois des ruches. Ann. Abeille 3, $85-98$.

(153) Gonnet M. el al, 1960. Influence du chauffage sur le facteur antibiotique présent dans les miels. Amn. Abeille, 3, 349-364.

(154) Gonnet M. el al., ig6o. Action antigerminative des produits de la ruche d'Abeilles (Apis nellifica). sur les graines et les tubercules. C.R. Acad. Sci., 250, 6r 2-6I 4.

(155) Lavie P., ig60. Les substances antibactériennes dans la colonie d'Abeilles (Apis mellifica L.). (Thèse. Paris).

(156) Lecomte J., Ig6o. Observations sur le comportement des Abeilles butineuses. Annt. Abeille, 3, 317-327.

(157) Malrizio A., et al. ig6o. Pollens de plantes mellifères d'Europe. Pollen et spores, 2, i59-182.

(I58) Naulleau G., I960. Les transpositions de larves de différentes castes d'Apis mellifica dans les cellules d'autres castes. Étude des types intermédiaires entre reine et ouvrières. Ann. Abeille, 3, 65-83.

(159) Pain J. et al., ig6o. Sur les constituants du mélanire attractif des glandes mandibulaires des reines d'Abeilles (Apis mellifica L.) à différents stades de leur vie. C. R. Acad. Sci., 251, 1046-1048.

(160) PAin J. el al., ig60. Mise en évidence d'une substance attractive extraite du corps des ouvrières d'abeilles non orphelines (Apis mellifica L.). C. R. Acal. Sci., 250, i r 26- I 27.

(i6r) Pain J., ig5o. De l'influence du nombre des Abeilles encagées sur la formation des oufs dans les ovaires de l'ouvrière. C. R. Acad. Sci., 250, 2639-263I.

(162) Ropartz P., ig6o. Sur les réactions des Abeilles à la lumière. Ann. Abeille, 3, 335-338.

(163) VuitaAume M. et al., I960. Construction de cellules rondes ct de cellules irrégulières chez Apis mellifica. Ann. Abeille, 3, 45-63. 